\title{
Fully self-consistent study of charge-exchange resonances and the impact on the symmetry energy parameters
}

\author{
X. Roca-Maza, ${ }^{1,2}$ Li-Gang Cao, ${ }^{3}$ G. Colò,${ }^{1,2}$ and H. Sagawa ${ }^{4,5}$ \\ ${ }^{1}$ Dipartimento di Fisica, Università degli Studi di Milano, via Celoria 16, Milano 20133, Italy \\ ${ }^{2}$ Istituto Nazionale di Fisica Nucleare (INFN), Sezione di Milano, via Celoria 16, Milano 20133, Italy \\ ${ }^{3}$ School of Mathematics and Physics, North China Electric Power University, Beijing 102206, China \\ ${ }^{4}$ RIKEN, Nishina Center, Japan \\ ${ }^{5}$ Center for Mathematics and Physics, University of Aizu, Fukushima 965-0001, Japan \\ (Received 26 July 2016; revised manuscript received 16 September 2016; published 17 October 2016)
}

\begin{abstract}
We have examined within a fully self-consistent theoretical framework the energy difference between the anti-analog giant dipole resonance (AGDR) and the isobaric analog state (IAS), $E_{\mathrm{AGDR}}-E_{\mathrm{IAS}}$, as an indicator of the neutron skin and of the density behavior of the symmetry energy. We have improved two specific points in our HF+RPA calculations: (1) the exchange term of the two-body Coulomb interaction is treated exactly without Slater approximation; and (2) the two-parameters spin-orbit interaction is treated in a consistent way within the energy density functional theory. The estimated values for the neutron skin in ${ }^{208} \mathrm{~Pb}$ and the slope parameter of symmetry energy are compared with previous analysis available in the literature.
\end{abstract}

DOI: 10.1103/PhysRevC.94.044313

\section{INTRODUCTION}

A comprehensive effort is currently being devoted to the determination of the nuclear symmetry energy, in particular as far as its density dependence is concerned. The symmetry energy is defined starting from the nuclear matter equation of state. The energy per particle in a uniform system characterized by a density $\rho$ and a relative neutron-proton asymmetry $\beta \equiv$ $\left(\rho_{n}-\rho_{p}\right) / \rho$ can be estimated by expanding up to second order in $\beta$ as

$$
\frac{E}{A}(\rho, \beta)=\frac{E}{A}(\rho, \beta=0)+S(\rho) \beta^{2} .
$$

This truncation has been found to be accurate for densities up to at least $2 \rho_{0}$, where $\rho_{0}$ is the saturation density [1]. Hence, the symmetry energy $S(\rho)$ can be seen as the difference between the energy per particle in neutron matter $(\beta=1)$ and symmetric matter $(\beta=0)$. Its value at saturation density is often defined as $J \equiv S\left(\rho_{0}\right)$. Expanding $S$ around $\rho_{0}$ in terms of $\left(\rho-\rho_{0}\right) / \rho_{0}$ leads to the definition of the well-known, and highly debated, slope parameter $L \equiv 3 \rho_{0} S^{\prime}\left(\rho_{0}\right)$. We recall that $L$ and the neutron skin thickness $\Delta r_{n p} \equiv\left\langle r^{2}\right\rangle_{n}^{1 / 2}-\left\langle r^{2}\right\rangle_{p}^{1 / 2}$ in a medium-heavy nucleus are strongly correlated [2-4]. Therefore, works aimed at extracting $L$ from various observables can also give an estimate for the neutron skin. Conversely, new measurements of the neutron skin have contributed to our understanding of the behavior of the symmetry energy. Examples are experiments on parity violating elastic electron scattering [5], elastic proton scattering [6,7], antiprotonic and pionic atoms [8,9], or coherent pion photoproduction [10].

Continuous efforts from both experimental and theoretical sides have narrowed significantly the possible range of values of $J$ and $L$. Review papers [11-14] and even a whole volume [15] have been devoted to such strenuous efforts. Note that nuclear structure studies, the analysis of nuclear collisions with different projectile combinations and different incident energies, as well as several astrophysical observations have complemented with one another and been all instrumental to reach the present status. A fair account of the overall conclusion (yet not final) of this quest can be given by quoting the numbers of Table 1 in Ref. [16]: the values that have been pointed out for $J$ and $L$ (with the average error bars) are $\approx 32 \mathrm{MeV}$ and $\approx 59 \mathrm{MeV}$, respectively (with an average error of $\approx 3 \mathrm{MeV}$ and $\approx 16 \mathrm{MeV}$, respectively).

Some of the observables which are sensitive to the symmetry energy have been extensively exploited. Within the nuclear structure realm, we have chiefly in mind isovector excitations like the isovector giant dipole resonance (IVGDR) [17], the isovector quadrupole resonance (IVGQR) [18], and the total dipole polarizability [19-21]. It is worth noting that several works in the literature [22-26] where the same observables have been independently analyzed provide actually consistent conclusions for $J$ and $L$. Masses can also of course give valuable indications on such quantities [27-29].

At the same time, either crosschecks or improvements of these findings can be obtained by exploiting the well-known concept of isospin symmetry. This was the spirit of Ref. [30], in which it has been argued that the mass formula can be extended to isobaric analog resonances, and these are more specifically sensitive to the symmetry energy. Along a similar line, one can look, rather than the energy of the GDR with respect to the ground state of nucleus $(N, Z)$, at the energy difference between the corresponding states in the nucleus $(N-1, Z+1)$. This has been the purpose of our previous work [31]. Therein, we have also presented a model that substantiates our intuition, that is, we have explicitly shown with a simplified yet realistic model that the energy difference $E_{\mathrm{AGDR}}-E_{\mathrm{IAS}}$ can be correlated with the neutron skin and at the same time with the slope parameter $L$ of the symmetry energy. This correlation has been shown to be fulfilled by microscopic calculations, although in a model-dependent way, and has allowed the extraction of the quantities of interest based on available experimental data.

Specifically, it is well known that the IAS is the analog of the mother ground state with $T=T_{0}$. On the other hand, the 
GDR in the daughter nucleus $T_{z}=T_{0}-1$ has three isospin components, analog $\left(T=T_{0}+1, T=T_{0}\right)$, and anti-analog ( $\left.T=T_{0}-1\right)$ components. However, in a nucleus with neutron excess the lowest isospin component is the dominant one. In fact, the transition probabilities of the three isospin components are weighted by the isospin factors $\left(2 T_{0}-1\right) /\left(2 T_{0}+1\right)$, $1 /\left(T_{0}+1\right)$, and $1 /\left(T_{0}+1\right)\left(2 T_{0}+1\right)$ in the cases, respectively, of $T=T_{0}-1, T_{0}$, and $T_{0}+1$. Consequently, in ${ }^{208} \mathrm{~Pb}$ the lowest component exhausts almost $98 \%$ of the GDR strength, and the GDR in the daughter nucleus can be identified with the so-called anti-analog $T_{0}-1$ component. We shall use the label AGDR in this work, as in the previous one, in order not to make any confusion with existing conventions.

Two experiments have been recently performed with the goal of measuring the energy difference $E_{\mathrm{AGDR}}-E_{\mathrm{IAS}}$ in ${ }^{208} \mathrm{~Pb}$ [32,33]. In our previous work, we have used both experimental results to pin down the constraint for the symmetry energy parameters. The results for $L$ and for the neutron skin have been found to be significantly larger than those previously obtained in the literature. Understanding the discrepancy between these results is the main motivation of our current work. To this aim, we have improved the theoretical analysis in several ways.

In this work, as in the previous Ref. [31] and in similar papers [34], the basic idea is to use nuclear energy density functionals (EDFs) that provide different values for $J, L$, and the neutron skin, and calculate the energy difference $E_{\mathrm{AGDR}}-E_{\mathrm{IAS}}$ in ${ }^{208} \mathrm{~Pb}$. After checking the existence of the above-mentioned linear correlations, one can employ the experimental value for the energy difference $E_{\mathrm{AGDR}}-E_{\mathrm{IAS}}$, and deduce the corresponding values of $J, L$, and the neutron skin. Proper consideration of the relevant sources of error is a big issue as we shall see below. In Refs. [24,31] a family of EDFs called SAMi-J has been introduced: they are fitted by using the same protocol of the original SAMi interaction [35]. They are used within the self-consistent charge-exchange random phase approximation (RPA) model, that is the tool of choice to calculate IAS and AGDR energies.

Here, we have improved in three ways in our self-consistent model and its analysis: (i) the two-body spin-orbit interaction had not been taken into account in Ref. [31], while we adopt its exact form in the present study; (ii) we have checked the effect of implementing the exact Coulomb exchange; and (iii) we have checked also the way to extract the energy centroid of the AGDR from the charge-exchange RPA calculations.

The structure of the paper is as follows. In Sec. II we discuss the theoretical framework. We of course refrain from repeating what has been discussed at length in Ref. [31], and we instead focus on the introduction of exact two-body spin-orbit matrix elements and exact Coulomb exchange. As we have stressed above, these points may be of interest in itself for the reader, as they may impact other kinds of nuclear structure research. New results for the AGDR energies, comparisons with previous ones, and consequences for the symmetry energy parameters are the subject of Sec. III. Finally, our conclusions are drawn in Sec. IV.

\section{THEORY FRAMEWORK}

The calculations whose results are reported below parallel those already reported in Ref. [31]. We shall not repeat here the discussion about the general charge-exchange RPA framework and the numerical details, that is already available in that work. We shall focus on the main differences between those calculations and the new ones, that are the proper implementation of the residual interaction associated with the two-parameter spin-orbit functional, and the inclusion of exact Coulomb exchange.

\section{A. Two parameter spin-orbit functional}

The functionals of the SAMi family $[24,35]$ include a spinorbit term of the form

$$
E_{\text {s.o. }}=\int d^{3} r\left(b_{4} \mathbf{J} \cdot \nabla \rho+b_{4}^{\prime} \sum_{q} \mathbf{J}_{q} \cdot \nabla \rho_{q}\right),
$$

where $\rho$ is the usual density, $\vec{J}$ is the spin-orbit density, and $q$ labels either protons or neutrons. This form, which is characterized by two parameters $b_{4}$ and $b_{4}^{\prime}$, has been first introduced in Ref. [36], and cannot be trivially derived from an effective Hamiltonian. Consequently, the two-body matrix elements of the associated residual RPA interaction have to be calculated by means of functional derivative (cf., e.g., p. 130 of Ref. [37]). The form of this interaction has been given in Refs. [38,39] in the case of non-charge-exchange excitations. In the case of charge-exchange excitations, the spin-orbit term can be found in Ref. [40] as

$$
\begin{aligned}
V & =-2 i\left(C_{0}^{\nabla J}+C_{1}^{\nabla J} \tau_{1} \tau_{2}\right) V_{L S}, \\
V_{L S} & \equiv\left(\sigma_{1}+\sigma_{2}\right) k^{\prime} \times \delta\left(r_{1}-r_{2}\right) k,
\end{aligned}
$$

where

$$
C_{0}^{\nabla J}=-b_{4}-\frac{b_{4}^{\prime}}{2}, \quad C_{1}^{\nabla J}=-\frac{b_{4}^{\prime}}{2} .
$$

We propose in our model an alternative consistent way to recover Eq. (2) from Eq. (3). This can be done by using non-antisymmetrized (NAS) matrix elements of Eq. (3). That is, if and only if one calculates the direct matrix element as

$$
E_{\mathrm{s} . \mathrm{o} .}=\frac{1}{2} \sum_{i j}\langle i j|V| i j\rangle_{\mathrm{NAS}},
$$

it can be shown that the matrix element reads back to Eq. (2). We first take into account an identity

$$
\begin{aligned}
-2 i\left(C_{0}^{\nabla J}+C_{1}^{\nabla J} \tau_{1} \tau_{2}\right) & =-2 i\left[C_{0}^{\nabla J}+C_{1}^{\nabla J}\left(2 P_{\tau}-1\right)\right] \\
& =-2 i\left[\left(C_{0}^{\nabla J}-C_{1}^{\nabla J}\right)+2 C_{1}^{\nabla J} P_{\tau}\right],
\end{aligned}
$$

where $P_{\tau}$ is the isospin exchange operator. We then write our force as

$$
V=-2\left(C_{0}^{\nabla J}-C_{1}^{\nabla J}\right) V_{L S}-4 C_{1}^{\nabla J} V_{L S} P_{\tau},
$$


so that we can use the well-known result of Appendix B of [41] to arrive at

$$
\begin{aligned}
E_{\mathrm{s} . \mathrm{o} .}= & -\left(C_{0}^{\nabla J}-C_{1}^{\nabla J}\right) \int d^{3} r \vec{\nabla} \rho \cdot \vec{J} \\
& -2 C_{1}^{\nabla J} \sum_{q} \int d^{3} r \vec{\nabla} \rho_{q} \cdot \vec{J}_{q} \\
= & b_{4} \int d^{3} r \vec{\nabla} \rho \cdot \vec{J}+b_{4}^{\prime} \sum_{q} \int d^{3} r \vec{\nabla} \rho_{q} \cdot \vec{J}_{q},
\end{aligned}
$$

which is indeed identical to Eq. (2).

In our previous work [31], we had not implemented the two-body spin-orbit in the residual interaction. As we shall see below, its implementation is important to ensure that selfconsistent RPA does properly restore the isospin symmetry, and has also an effect on the energy centroids.

\section{B. Coulomb exchange}

In connection with the Skyrme interaction, it is customary to adopt the so-called Slater approximation for the Coulomb exchange. The Slater approximation is local and, hence, it does not break the assumption of zero-range; thus, it allows the use of simpler codes when working in coordinate space. The HF equation for protons includes the effect of the direct and exchange Coulomb field on the wave function. The direct part reads

$$
U_{\mathrm{C}}^{\mathrm{dir} .}(\vec{r}) \varphi_{i}(\vec{r})=\frac{e^{2}}{2} \int d^{3} r^{\prime} \frac{\rho_{p}\left(\vec{r}^{\prime}\right)}{\left|\vec{r}-\vec{r}^{\prime}\right|} \varphi_{i}(\vec{r})
$$

and the exchange part is also local within the Slater approximation,

$$
U_{\mathrm{C}}^{x, \text { Slater }} \varphi_{i}(\vec{r})=-\frac{e^{2}}{2}\left(\frac{3}{\pi}\right)^{1 / 3} \rho_{p}^{1 / 3} \varphi_{i}(\vec{r}),
$$

where $e$ is the elementary electric charge, $\rho_{p}$ is the proton density, and $\varphi_{i}$ is the single particle wave function of the $i$ th nucleon. The exact exchange has instead the well-known nonlocal form,

$$
U_{\mathrm{C}}^{x, \text { exact }} \varphi_{i}(\vec{r})=-\frac{e^{2}}{2} \int d^{3} r^{\prime} \frac{\varphi_{j}^{*}\left(\vec{r}^{\prime}\right) \varphi_{j}(\vec{r})}{\left|\vec{r}-\vec{r}^{\prime}\right|} \varphi_{i}\left(\vec{r}^{\prime}\right)
$$

Since the early pioneering calculations done with the Skyrme force, the Slater approximation has been known to work quite well. In particular, the relative difference between the exact total energy and that calculated with the Slater approximation has been found to be of the order of 3-6\% in Ref. [42]. Thus, we may expect that the effects arising from the replacement of the Slater approximation by the exact exchange can be treated in a perturbative prescription described below. Our strategy is that we replace the single particle energies with those calculated with exact Coulomb matrix elements, but do not change the HF wave functions calculated by the Slater approximation (see the Appendix for details). While the Coulomb interaction contributes to the HF field, the Coulomb residual interaction have no contribution to charge-exchange RPA matrix elements.
In practice, the HF equation has been firstly solved within the Slater approximation,

$$
\left[H+U_{\mathrm{C}}^{\text {dir. }}+U_{\mathrm{C}}^{x, \text { Slater }}\right] \varphi_{i}=\varepsilon_{i} \varphi_{i} .
$$

Then, in a second step, the single-particle energies $\varepsilon_{i}$ have been corrected perturbatively, namely, new energies $\tilde{\varepsilon}_{i}$ have been extracted from

$$
\tilde{\varepsilon}_{i}=\varepsilon_{i}+\int d^{3} r \varphi_{i}^{*}(\vec{r})\left(U_{\mathrm{C}}^{x, \text { exact }}-U_{\mathrm{C}}^{x, \text { Slater }}\right) \varphi_{i}(\vec{r}) .
$$

As we shall see below, the single-particle energies undergo small changes due to the correction $\Delta U \equiv U_{\mathrm{C}}^{x \text {,exact }}-U_{\mathrm{C}}^{x \text {,Slater }}$, that is, at most $\approx 500 \mathrm{keV}$ (cf. Table II). Thus, considering the wave functions as unchanged is a fairly good assumption. We, therefore, will perform RPA with exact Coulomb exchange by just replacing proton single-particle energies $\varepsilon_{i}$ with their new values $\tilde{\varepsilon}_{i}$.

\section{RESULTS}

\section{A. Testing the two-body spin-orbit residual force}

We have tested the RPA charge-exchange code, and in particular the implementation of the two-body spin-orbit residual force, by running it without the Coulomb interaction. It is well known that a fully self-consistent RPA restores the symmetries that are broken at the HF level, such as the isospin symmetry. In the case of no Coulomb interaction, we should obtain a single IAS peak at zero energy that exhausts the full sum rule value strength $N-Z$ due to the restoration of isospin symmetry. In Table I we report the results we have obtained for the test case of ${ }^{90} \mathrm{Zr}$ by switching off the Coulomb force. The IAS is very close to zero, as its energy never exceeds $8 \mathrm{keV}$. The strength is essentially $N-Z$ in all cases, and no systematic deviation is observed. This is the best proof of the numerical accuracy of our calculations.

In Table I, we also report the unperturbed energy difference $\Delta \varepsilon$ between the proton and neutron $1 g_{9 / 2}$ states that corresponds to the dominant transition in the IAS of ${ }^{90} \mathrm{Zr}$, the associated diagonal matrix element $V_{p h}$ and their sum.

TABLE I. Energy of the IAS, $E_{\mathrm{IAS}}$, and strength $S$ calculated in ${ }^{90} \mathrm{Zr}$ by dropping the Coulomb term in the Hamiltonian. We also report the unperturbed energy difference $\Delta \varepsilon$ between the proton and neutron $1 g_{9 / 2}$ states, the corresponding diagonal matrix element $V_{p h}$ and their sum.

\begin{tabular}{lcrrcc}
\hline \hline Force & $\begin{array}{c}E_{\mathrm{IAS}} \\
{[\mathrm{MeV}]}\end{array}$ & \multicolumn{1}{c}{$S$} & $\begin{array}{c}\Delta \varepsilon \\
{[\mathrm{MeV}]}\end{array}$ & $\begin{array}{c}V_{p h} \\
{[\mathrm{MeV}]}\end{array}$ & $\begin{array}{c}\Delta \varepsilon+V_{p h} \\
{[\mathrm{MeV}]}\end{array}$ \\
\hline SAMi-J27 & 0.008 & 9.99 & -4.61 & 5.39 & 0.78 \\
SAMi-J28 & 0.005 & 10.00 & -4.86 & 5.44 & 0.58 \\
SAMi-J29 & 0.003 & 10.00 & -4.99 & 5.41 & 0.42 \\
SAMi-J30 & 0.002 & 10.00 & -5.05 & 5.40 & 0.34 \\
SAMi-J31 & 0.002 & 9.99 & -5.06 & 5.39 & 0.33 \\
SAMi-J32 & 0.002 & 9.99 & -5.03 & 5.41 & 0.37 \\
SAMi-J33 & 0.003 & 9.99 & -4.97 & 5.42 & 0.45 \\
SAMi-J34 & 0.004 & 9.99 & -4.91 & 5.45 & 0.54 \\
SAMi-J35 & 0.006 & 9.99 & -4.84 & 5.48 & 0.63 \\
\hline \hline
\end{tabular}


TABLE II. Single-particle proton levels in ${ }^{208} \mathrm{~Pb}$ calculated with the SAMi interaction. Results obtained with the exact Coulomb exchange, introduced as explained in the text, are compared with those obtained with the Slater approximation.

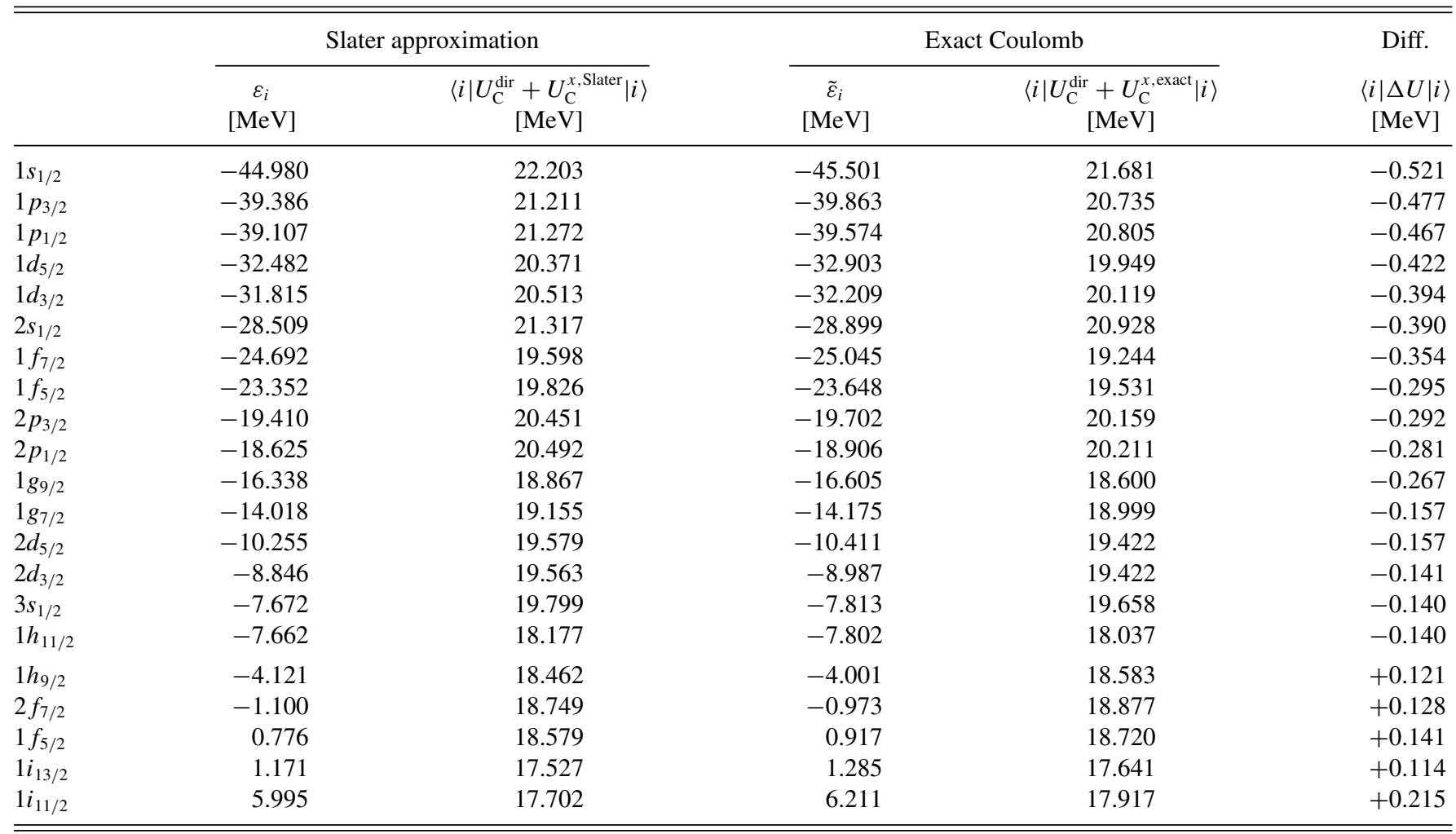

Although this is the main component of the IAS wave function, the unperturbed energy and the $p$ - $h$ matrix element cancel out only at the level of hundreds of $\mathrm{keV}$, and not at the $\mathrm{keV}$ scale. This means that the whole model space is needed to ensure the proper behavior of RPA, due also to the zero-range nature of the force.

In the case of the full calculations, including the Coulomb interaction, for ${ }^{208} \mathrm{~Pb}$, the implementation of the exact twobody residual force has a non-negligible effect as we shall discuss below.

\section{B. The exact Coulomb exchange}

The effect of the Coulomb exchange on the proton levels of ${ }^{208} \mathrm{~Pb}$ can be seen in Table II in the case of the SAMi interaction. Nonetheless, we have seen that the results obtained with the other interactions of the SAMi-J family are quite similar to the present ones.

The single-particle levels are moved by hundreds of $\mathrm{keV}$ by the exact Coulomb exchange. With shifts of this order, the wave functions are not expected to vary and the choice of using the perturbative treatment seems to be well justified. The Coulomb exchange has an attractive effect on proton levels, that counteracts partly the direct Coulomb repulsion. For bound states, the Slater approximation underestimates this effect, but the difference between exact exchange and approximate exchange tends to decrease when approaching the Fermi energy. This is quite natural, since the Slater approximation identifies the exchange potential with the one generated by a free particle gas with the same average density [43], and the wave functions close to the Fermi energy are more distributed in space and more likely to sample an averaged exchange potential. For bound and quasibound states above the Fermi energy the Slater approximation provides instead slightly more attraction than the exact exchange, although by only $\approx 100 \mathrm{keV}$. These features are entirely due to the nature of Slater approximation in which the relative distance in Eq. (9) between interacting particles is replaced by a constant value appropriate to those around the Fermi level. Thus, the Slater approximation gives smaller contributions to hole states below the Fermi level when compared to exact calculations, but larger for particle states above the Fermi level.

In charge-exchange RPA, for a nucleus having neutron excess like ${ }^{208} \mathrm{~Pb}$, the $t_{-}$and $t_{+}$channels are rather decoupled. In the channel of interest here, namely $t_{-}$, the excited states are expected to feel the consequence of unoccupied proton levels being pushed upwards. In fact, the IAS energy increases by about $100 \mathrm{keV}$ or so, when the exact exchange is implemented. The same could happen for other charge-exchange states. The calculated IAS energies are shown in Fig. 1 as a function of neutron skin in ${ }^{208} \mathrm{~Pb}$. We can see clearly that the exact treatment of Coulomb exchange raises the IAS energy by more than $100 \mathrm{keV}$ making it closer to the experimental value and the DD-ME results [44] compared with that of Slater approximation. We can see also a variation of IAS energy for different size of neutron skin while the variation of Coulomb energy is around $100 \mathrm{keV}$ among different SAMi-J interaction. This trend is the same in the relativistic model DD-ME. 


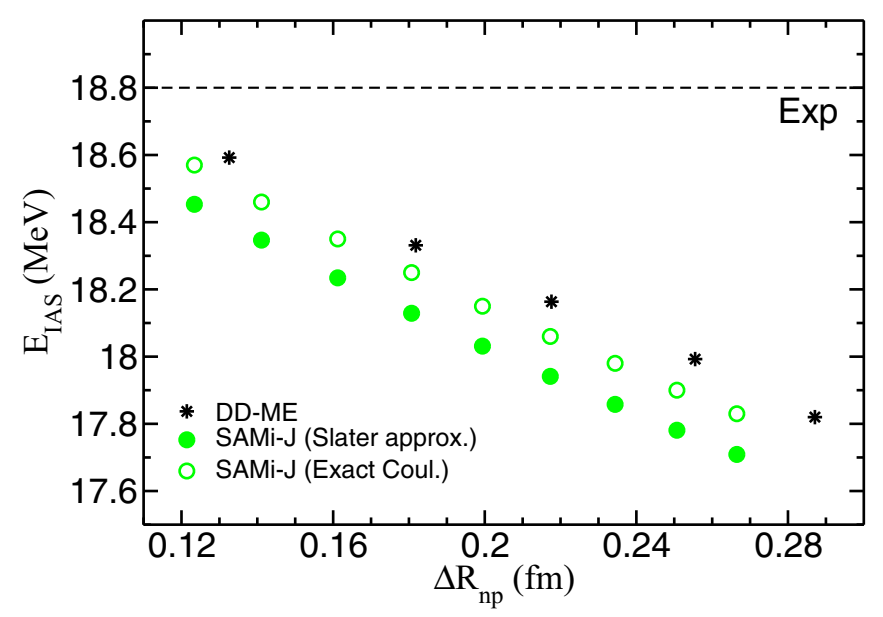

FIG. 1. The energy of the IAS as a function of neutron skin in ${ }^{208} \mathrm{~Pb}$ calculated with SAMi-J interactions with the exact Coulomb exchange (empty circles) and the Slater approximation (full circles). We also display (stars) the results obtained with the covariant DD-ME Lagrangians [44].

We would like to raise two open problems in the IAS study with microscopic models: (1) while the charge radius of ${ }^{208} \mathrm{~Pb}$ is properly obtained by the calculations, within a $0.2 \%$ spread with respect to the optimal value of $5.50 \mathrm{fm}$ predicted by the SAMi interaction [35], the calculated IAS energy is still lower than the experimental observation by 200-900 keV; (2) a variation of about $1 \mathrm{MeV}$ of the IAS energy among different parameter sets is observed, in both SAMi-J and DD-ME calculations, while the charge radius is only slightly changed (by $0.2 \%$ at most in the case of SAMi-J). These two problems should be addressed in a future study with more stringent constrain for the proton density profile together with the charge radius in a self-consistent microscopic theory.

\section{Difference between AGDR and IAS energies and the impact on the symmetry energy and neutron skin}

In this subsection, we display some of the results that have been already presented in Ref. [31], and we discuss how and why they have changed by the present new calculations. The most important outcome is displayed in Fig. 2. The experimental results and the theoretical results obtained by means of relativistic mean field (RMF) calculations with the DD-ME effective Lagrangian introduced in Ref. [34], are obviously the same as in Fig. 4 of [31]. The introduction of the exact two-body spin-orbit has changed the SAMi-J results in absolute value (by about 200-400 keV), and has induced a change in the correlation between $E_{\mathrm{AGDR}}-E_{\mathrm{IAS}}$ and the neutron skin thickness. Specifically, the correlation coefficient is now 0.9996 and the slope of the linear fit is $-9.8 \mathrm{MeV} / \mathrm{fm}$ instead of 0.997 and $-5.5 \mathrm{MeV} / \mathrm{fm}$, respectively, of our previous calculation. As we have mentioned, we have also tried to identify the AGDR centroid in a more precise way than it has been done before: such centroid has been here defined in the region where the strength is larger than $10 \%$ of the value associated with the height of the AGDR peak. We also note that this provides an energy range as wide as the experimental

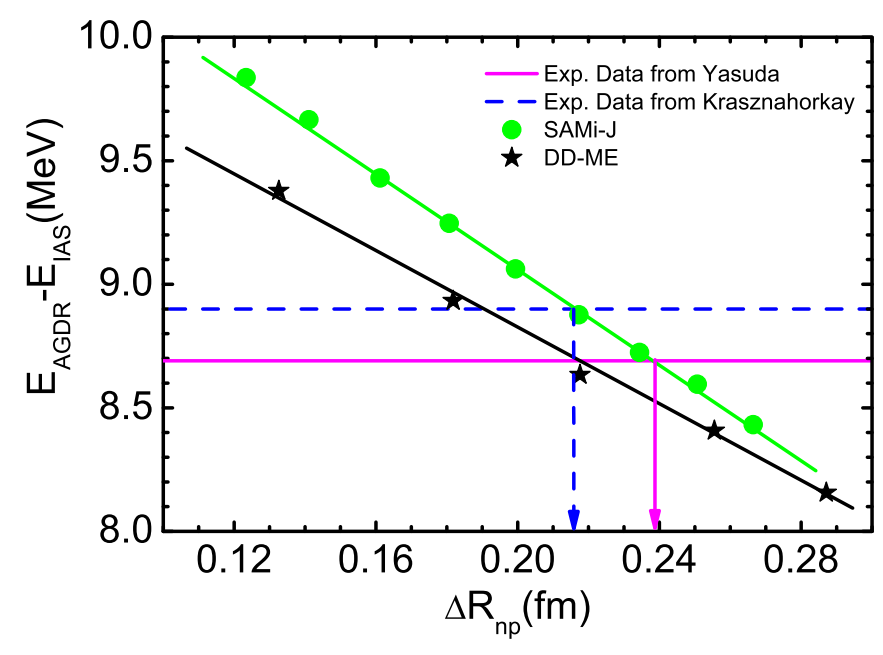

FIG. 2. The energy difference $E_{\mathrm{AGDR}}-E_{\mathrm{IAS}}$ of AGDR and IAS as a function of the neutron-skin thickness, obtained by using the SAMi-J family of Skyrme functionals. The calculated values are presented as solid circles. Two different experimental data $[32,33]$ are also shown as solid (magenta) and dashed (blue) lines, respectively. The arrows indicate the neutron skin constrained by these experimental data. We also display results obtained with the covariant DD-ME Lagrangians of Ref. [32].

one although slightly shifted. Finally, the Coulomb exchange impacts the IAS and AGDR peaks because of the shift of the unoccupied proton states that has been illustrated in the previous subsection; but since the two peaks are affected in a similar way, no significant shift of $E_{\mathrm{AGDR}}-E_{\mathrm{IAS}}$ is produced.

By comparing Fig. 2 with Fig. 4 of our previous work, one can see that the predictions for the neutron skin are moved down. The effect is tiny in the case of the value deduced from the experimental data of Krasznahorkay et al. from Ref. [32], i.e., the change is from $0.218 \pm 0.015 \mathrm{fm}$ to $0.216 \pm 0.010 \mathrm{fm}$. On the other hand, it is larger if we adopt the experimental data by Yasuda et al. from Ref. [33]: the neutron skin is moved down from $0.254 \pm 0.062 \mathrm{fm}$ to $0.239 \pm 0.038 \mathrm{fm}$. We recall that, consistently with our previous work [31], the reported errors are just propagated from the experiment. That is, no systematic errors or theoretical errors have been assessed here.

This triggers, in turn, an effect on the deduced values of $J$ and $L$ within our model as highlighted in Fig. 3. If we compare this figure with Fig. 5 of [31], we observe that the predictions for $J$ and $L$ are shifted towards lower values as well. By considering the data from [32] our prediction for $J$ moves down from $32.2 \pm 0.9 \mathrm{MeV}$ to $32.0 \pm 0.6 \mathrm{MeV}$, while by considering the data from [33] we move down from $34.2 \pm$ $3.5 \mathrm{MeV}$ to $33.3 \pm 2.1 \mathrm{MeV}$. Concerning the slope parameter, by using the data from [32] our prediction for $L$ moves down from $86.1 \pm 9.1 \mathrm{MeV}$ to $85.3 \pm 5.9 \mathrm{MeV}$, whereas by using the data from [33] we move down from $108.5 \pm 35.8 \mathrm{MeV}$ to $98.8 \pm 23.6 \mathrm{MeV}$. As above, the changes are small if the data of Ref. [32] is used, but are significantly larger (up to $\approx 10 \%$ on $L$ ) if that of Ref. [33] is used. This goes in the direction of reconciling to some extent our results with those available in the existing literature. 

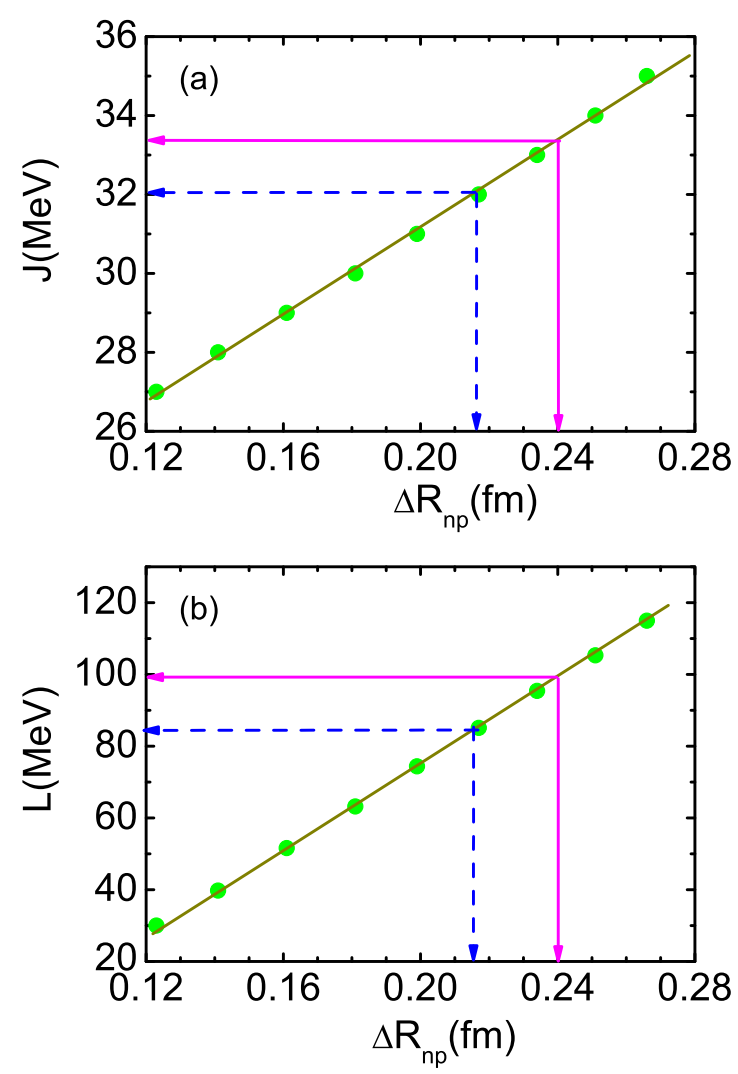

FIG. 3. (a) and (b) show the correlations between the neutron-skin thickness and either the symmetry energy $J$ at saturation density or the corresponding slope parameter $L$, respectively. The constraints provided by the experimental data already shown in Fig. 2.

In Ref. [31] we had chosen to report weighted averages of the results obtained from the two experiments. Clearly, the error bars that we have reported only correspond to the propagation of the experimental error bars. The possible systematic errors are not taken into account as well as the theoretical uncertainties associated with the model-dependent extraction of the results for $J, L$, and the neutron skin thickness. In this respect, these are lowest-limit estimates of the possible errors and one should take weighted averages, in which the error further decreases, with great care. In the current work we prefer to report the whole range covered by the estimates of $J, L$, and the neutron skin when both experiments are considered. Such ranges are shown in Table III.

TABLE III. Allowed range for $J, L$, and the neutron skin when both experimental data from [32,33] are considered. The estimates from the previous theoretical calculations of Ref. [31] and from the current work are compared with each other.

\begin{tabular}{lccc}
\hline \hline & & Ref. [31] & Present work \\
\hline$J$ & Allowed range & $30.7-37.7 \mathrm{MeV}$ & $31.2-35.4 \mathrm{MeV}$ \\
$L$ & Allowed range & $72.7-144.3 \mathrm{MeV}$ & $75.2-122.4 \mathrm{MeV}$ \\
$\Delta R_{n p}$ & Allowed range & $0.192-0.316 \mathrm{fm}$ & $0.201-0.277 \mathrm{fm}$ \\
\hline \hline
\end{tabular}

\section{CONCLUSIONS}

We have studied within a fully self-consistent theoretical framework the energy difference between the anti-analog giant dipole resonance (AGDR) and the isobaric analog state, $E_{\mathrm{AGDR}}-E_{\mathrm{IAS}}$, as an indicator of the neutron skin and of the density behavior of the symmetry energy. In the present study, we have improved in three ways the fully self-consistent model and its analysis: (i) while the two-body spin-orbit interaction had not been considered in Ref. [31], we adopt the two-parameter form of spin-orbit interaction in the present calculations. It has a profound effect on the restoration of the isospin symmetry in the HF+RPA results. The centroid energies of ADGR and IAS are also affected by about 200 $400 \mathrm{keV}$ and the correlation and slope of the linear fit between the difference $E_{\mathrm{ADGR}}-E_{\mathrm{IAS}}$ and the neutron skin are changed; (ii) we have checked the effect of implementing the exact Coulomb exchange in charge-exchange HF+RPA. The effect on the IAS is of shifting up the energy by more than $100 \mathrm{keV}$ and making the calculations closer to the experimental value as compared to the case in which the Slater approximation is adopted. On the other hand, the effects on the AGDR and IAS centroids tend to cancel out and be small in the final results; (iii) we have checked also the way to extract the energy centroid of the AGDR from the charge-exchange RPA calculations. However, this does not have a profound effect on the results.

The final results for the symmetry energy parameters $J$ and $L$ are changed by $10 \%$ at most and tend to be slightly closer to the empirical values obtained by other analysis in the literature. There are still open problems, such as how to obtain a better agreement with the experimental energy of IAS in ${ }^{208} \mathrm{~Pb}$, and the strong correlation of the IAS energy with the neutron skin, in which the variation of the IAS energy is larger than expected from the change of the charge radii within the SAMi-J family.

\section{ACKNOWLEDGMENTS}

We are indebted to N. Paar for useful discussions and for providing us with the calculations for the IAS presented for the first time in this work for the DD-ME functional. This work is partly supported by the National Natural Science Foundation of China under Grants No. 11575060, No. 11175216, and No. 11435014, and the Fundamental Research Funds for the Central Universities (JB2014241). Funding from the European Union's Horizon 2020 research and innovation program under grant Agreement No. 654002 is also acknowledged.

\section{APPENDIX: EXACT COULOMB EXCHANGE ENERGY}

The exact Coulomb exchange energy is calculated by the perturbative method described in the main text. Here, we provide some more detailed information together with the formulas that have been input in our code. First, the HartreeFock (HF) single-particle wave functions are calculated by taking a conventional treatment of the Coulomb exchange term in the HF field, i.e., the Slater approximation. Then, the exact Coulomb exchange field of Eq. (9) is calculated, together with the exact Coulomb exchange contribution to the total binding energy. This latter is of course obtained from the sum of the matrix elements associated with the exact exchange interaction 
energy for two-particle configurations:

$$
\int d \vec{r} \int d \vec{r}^{\prime} \varphi_{1}^{*}(\vec{r}) \varphi_{2}(\vec{r}) \frac{e^{2}}{\left|\vec{r}-\overrightarrow{r^{\prime}}\right|} \varphi_{2}^{*}\left(\vec{r}^{\prime}\right) \varphi_{1}\left(\vec{r}^{\prime}\right) .
$$

In Eqs. (9) and (A1), the Coulomb interaction can be decomposed as

$$
\begin{aligned}
\frac{e^{2}}{\left|\vec{r}-\overrightarrow{r^{\prime}}\right|} & =e^{2} \sum_{l} P_{l}(\theta) \frac{r_{<}^{l}}{r_{>}^{l+1}} \\
& =e^{2} \sum_{l m} \frac{4 \pi}{2 l+1} Y_{l m}^{*}\left(\hat{r^{\prime}}\right) Y_{l m}(\hat{r}) \frac{r_{<}^{l}}{r_{>}^{l+1}},
\end{aligned}
$$

where $P_{l}(\theta)$ is the Legendre polynomial, $Y_{l m}(\hat{r})$ are spherical harmonics and the radial variables $r_{>}$and $r_{<}$are defined to be $r_{>}=r$, and $r_{<}=r^{\prime}$ for $r>r^{\prime}$ and $r_{>}=r^{\prime}$ and $r_{<}=r^{\prime}$ for $r>r$ for $r^{\prime}>r$. The HF single-particle wave function is expressed as

$$
\varphi(\vec{r})=\frac{R_{l j}(r)}{r} Y_{l j m}(\hat{r}, \sigma),
$$

where $Y_{l j m}(\hat{r}, \sigma)$ is the spherical vector harmonics.

The Coulomb exchange energy (A1) for the two particle orbits $(1,2)$ is expressed in a compact form,

$$
\begin{aligned}
& \sum_{l} \frac{e^{2}\left\langle j_{1} 1 / 2 l 0 \mid j_{2} 1 / 2\right\rangle^{2}}{2 j_{2}+1}\left[\int_{0}^{\infty} d r \int_{0}^{r} d r^{\prime} \frac{\left(r^{\prime}\right)^{l}}{r^{l+1}}\right. \\
& \left.\quad+\int_{0}^{\infty} d r \int_{r}^{\infty} d r^{\prime} \frac{r^{l}}{\left(r^{\prime}\right)^{l+1}}\right] R_{l_{1} j_{1}}(r) R_{l_{2} j_{2}}\left(r^{\prime}\right) R_{l_{1} j_{1}}\left(r^{\prime}\right) R_{l_{2} j_{2}}(r),
\end{aligned}
$$

in which the Clebsch-Gordon coefficient is used.
[1] I. Vidaña, C. Providencia, A. Polls, and A. Rios, Phys. Rev. C 80, 045806 (2009).

[2] B. A. Brown, Phys. Rev. Lett. 85, 5296 (2000).

[3] R. Furnstahl, Nucl. Phys. A 706, 85 (2002).

[4] X. Roca-Maza, M. Centelles, X. Viñas, and M. Warda, Phys. Rev. Lett. 106, 252501 (2011).

[5] S. Abrahamyan, Z. Ahmed, H. Albataineh, K. Aniol, D. S. Armstrong, W. Armstrong, T. Averett, B. Babineau, A. Barbieri, V. Bellini et al. (PREX Collaboration), Phys. Rev. Lett. 108, 112502 (2012).

[6] G. W. Hoffmann, L. Ray, M. Barlett, J. McGill, G. S. Adams, G. J. Igo, F. Irom, A. T. M. Wang, C. A. Whitten, R. L. Boudrie et al., Phys. Rev. C 21, 1488 (1980).

[7] J. Zenihiro, H. Sakaguchi, T. Murakami, M. Yosoi, Y. Yasuda, S. Terashima, Y. Iwao, H. Takeda, M. Itoh, H. P. Yoshida et al., Phys. Rev. C 82, 044611 (2010).

[8] B. Klos, A. Trzcinska, J. Jastrzebski, T. Czosnyka, M. Kisielinski, P. Lubinski, P. Napiorkowski, L. Pienkowski, F. J. Hartmann, B. Ketzer et al., Phys. Rev. C 76, 014311 (2007).

[9] E. Friedman, Hyperfine Interact. 193, 33 (2009).

[10] C. M. Tarbert, D. P. Watts, D. I. Glazier, P. Aguar, J. Ahrens, J. R. M. Annand, H. J. Arends, R. Beck, V. Bekrenev, B. Boillat et al. (Crystal Ball at MAMI and A2 Collaboration), Phys. Rev. Lett. 112, 242502 (2014).

[11] B.-A. Li, L.-W. Chen, and C. M. Ko, Phys. Rep. 464, 113 (2008).

[12] M. B. Tsang, J. R. Stone, F. Camera, P. Danielewicz, S. Gandolfi, K. Hebeler, C. J. Horowitz, J. Lee, W. G. Lynch, Z. Kohley et al., Phys. Rev. C 86, 015803 (2012).

[13] J. M. Lattimer and Y. Lim, Astrophys. J. 771, 51 (2013).

[14] C. J. Horowitz, E. F. Brown, Y. Kim, W. G. Lynch, R. Michaels, A. Ono, J. Piekarewicz, M. B. Tsang, and H. H. Wolter, J. Phys. G: Nucl. Part. Phys. 41, 093001 (2014).

[15] B.-A. Li, À. Ramos, G. Verde, and I. Vidaña, Eur. Phys. J. A 50, 9 (2014).

[16] B.-A. Li and X. Han, Phys. Lett. B 727, 276 (2013).

[17] B. L. Berman and S. C. Fultz, Rev. Mod. Phys. 47, 713 (1975).

[18] S. S. Henshaw, M. W. Ahmed, G. Feldman, A. M. Nathan, and H. R. Weller, Phys. Rev. Lett. 107, 222501 (2011).
[19] A. Tamii, I. Poltoratska, P. von Neumann-Cosel, Y. Fujita, T. Adachi, C. A. Bertulani, J. Carter, M. Dozono, H. Fujita, K. Fujita et al., Phys. Rev. Lett. 107, 062502 (2011).

[20] D. M. Rossi, P. Adrich, F. Aksouh, H. Alvarez-Pol, T. Aumann, J. Benlliure, M. Böhmer, K. Boretzky, E. Casarejos, M. Chartier et al., Phys. Rev. Lett. 111, 242503 (2013).

[21] T. Hashimoto, A. M. Krumbholz, P.-G. Reinhard, A. Tamii, P. von Neumann-Cosel, T. Adachi, N. Aoi, C. A. Bertulani, H. Fujita, Y. Fujita et al., Phys. Rev. C 92, 031305(R) (2015).

[22] L.-G. Cao and Z.-Y. Ma, Chin. Phys. Lett. 25, 1625 (2008).

[23] L. Trippa, G. Colò, and E. Vigezzi, Phys. Rev. C 77, 061304 (2008)

[24] X. Roca-Maza, M. Brenna, B. K. Agrawal, P. F. Bortignon, G. Colò, L.-G. Cao, N. Paar, and D. Vretenar, Phys. Rev. C 87, 034301 (2013).

[25] X. Roca-Maza, M. Brenna, G. Colò, M. Centelles, X. Viñas, B. K. Agrawal, N. Paar, D. Vretenar, and J. Piekarewicz, Phys. Rev. C 88, 024316 (2013).

[26] X. Roca-Maza, X. Viñas, M. Centelles, B. K. Agrawal, G. Colò, N. Paar, J. Piekarewicz, and D. Vretenar, Phys. Rev. C 92, 064304 (2015).

[27] B. K. Agrawal, J. N. De, S. K. Samaddar, G. Colò, and A. Sulaksono, Phys. Rev. C 87, 051306(R) (2013).

[28] P. Moller, A. Sierk, T. Ichikawa, and H. Sagawa, At. Data Nucl. Data Tables 109-110, 1 (2016).

[29] P. Möller, W. D. Myers, H. Sagawa, and S. Yoshida, Phys. Rev. Lett. 108, 052501 (2012).

[30] P. Danielewicz and J. Lee, Nucl. Phys. A 922, 1 (2014).

[31] L.-G. Cao, X. Roca-Maza, G. Colò, and H. Sagawa, Phys. Rev. C 92, 034308 (2015).

[32] A. Krasznahorkay, M. Csatlós, L. Csige, T. K. Eriksen, F. Giacoppo, A. Görgen, T. W. Hagen, M. N. Harakeh, R. Julin, P. Koehler et al., arXiv:1311.1456.

[33] J. Yasuda, T. Wakasa, M. Okamoto, M. Dozono, K. Hatanaka, M. Ichimura, S. Kuroita, Y. Maeda, T. Noro, Y. Sakemi et al., Prog. Theor. Exp. Phys. (2013) 063D02.

[34] A. Krasznahorkay, N. Paar, D. Vretenar, and M. Harakeh, Phys. Lett. B 720, 428 (2013).

[35] X. Roca-Maza, G. Colò, and H. Sagawa, Phys. Rev. C 86, 031306(R) (2012). 
[36] P.-G. Reinhard and H. Flocard, Nucl. Phys. A 584, 467 (1995).

[37] M. Bender, P.-H. Heenen, and P.-G. Reinhard, Rev. Mod. Phys. 75, 121 (2003).

[38] J. Terasaki, J. Engel, M. Bender, J. Dobaczewski, W. Nazarewicz, and M. Stoitsov, Phys. Rev. C 71, 034310 (2005).

[39] G. Colo, L. Cao, N. V. Giai, and L. Capelli, Comput. Phys. Commun. 184, 142 (2013).
[40] M. Bender, J. Dobaczewski, J. Engel, and W. Nazarewicz, Phys. Rev. C 65, 054322 (2002).

[41] D. Vautherin and D. M. Brink, Phys. Rev. C 5, 626 (1972).

[42] C. Titin-Schnaider and P. Quentin, Phys. Lett. B 49, 397 (1974).

[43] J. C. Slater, Phys. Rev. 81, 385 (1951).

[44] N. Paar (private communication). 